PROCEEDINGS OF THE

AMERICAN MATHEMATICAL SOCIETY

Volume 124, Number 9, September 1996

\title{
FINITE CW COMPLEXES WITH MAXIMAL TORSION GAPS
}

\author{
HUALE HUANG
}

(Communicated by Thomas Goodwillie)

\begin{abstract}
We investigate some properties of finite CW complexes with maximal homotopy torsion gaps and prove a revision of the Halperin conjecture under an additional condition.
\end{abstract}

\section{INTRODUCTION}

Let $X$ be a simply connected finite CW complex and $n_{X}=\max \left\{i \mid H_{i}(X, Q) \neq 0\right\}$. On the other hand, $\pi_{i}(X)$ is the direct sum of finitely many copies of $\mathbb{Z}$ and a finite abelian group. We call an interval $[k, l]$ a torsion gap for $X$ if $\pi_{k}(X)$ and $\pi_{l}(X)$ both contain copies of $\mathbb{Z}$ and $\pi_{i}(X)(k<i<l)$ is finite. In [4], S. Halperin proved

Theorem 1. Let $X$ be a simply connected finite $C W$ complex. If $[k, l]$ is a torsion gap for $X$, then $l-k<n_{X}$.

Then, Halperin proposed the

Conjecture. If a simply connected finite $C W$ complex $X$ has at least one torsion gap of the form $\left[k, k+n_{X}-1\right]$, then $X$ has the rational homotopy type of a wedge of $n_{X}$-spheres.

In [6], the author resolved the conjecture in the negative. However, those CW complexes with maximal torsion gaps are very strict. In fact, we have

Theorem A. If a simply connected finite $C W$ complex $X$ has at least one torsion gap of the form $\left[k, k+n_{X}-1\right]$, then the $k$-connected covering $X(k)$ of $X$ has the rational homotopy type of a wedge of spheres.

Corollary 1. If $X$ has infinitely many torsion gaps of the form $\left[k, k+n_{X}-1\right]$, let $k_{0}=\min \left\{k \mid\left[k, k+n_{X}-1\right]\right.$ is a torsion gap for $\left.X\right\}$. Then if $[k, l]$ is a torsion gap for $X$ and $k \geq k_{0}$, then $l=k+n_{X}-1$ and $k \equiv 1\left(\bmod n_{x}-1\right)$. In particular, if all the torsion gaps for $X$ have the form $\left[k, k+n_{X}-1\right]$, then $X$ has the rational homotopy type of a wedge of $n_{X}$-spheres.

Proof. Since $\left[k_{0}, k_{0}+n_{X}-1\right]$ is a torsion gap for $X$, the $k_{0}$-connected covering $X\left(k_{0}\right)$ of $X$ is $\left(k_{0}+n_{X}-2\right)$-connected. By Theorem 1, there exists $m \leq n_{X}-1$, s.t. $\pi_{k_{0}+n_{X}-1+m}(X) \supset \mathbb{Z}$. As $k_{0} \geq 2,\left(k_{0}, n_{X}-1+m\right)+1<2\left(k_{0}+n_{X}-1\right)$; hence

$$
H^{k_{0}+n_{X}-1}\left(X\left(k_{0}\right), Q\right) \neq 0, \quad H^{k_{0}+n_{X}-1+m}\left(X\left(k_{0}\right), Q\right) \neq 0 .
$$

Received by the editors August 24, 1994 and, in revised form, January 19, 1995.

1991 Mathematics Subject Classification. Primary 55P62.

(C)1996 American Mathematical Society 
By Theorem A, $X\left(k_{0}\right)$ has the rational homotopy type of a wedge of spheres. So we have

$$
X\left(k_{0}\right)_{Q} \cong\left(S^{k_{0}+n_{X}-1} \vee S^{k_{0}+n_{X}-1+m} \vee Y\right)_{Q}
$$

Let $d=\left(k_{0}+n_{X}-2, k_{0}+n_{X}-2+m\right)$; then $d \mid m$. Then there is some integer $l$ s.t. for all $k \geq 0, \pi_{l+k d}(X) \supset \mathbb{Z}$. Hence $d=m=n_{X}-1$, and $k_{0} \equiv 1\left(\bmod n_{X}-1\right)$. Using the same argument, we can prove that if $[k, l]$ is a torsion gap for $X$ and $k \geq k_{0}$, then $l=k+n_{X}-1$ and $k \equiv 1\left(\bmod n_{X}-1\right)$.

As a revision of the Halperin conjecture, we have

Theorem B. If a simply connected finite $C W$ complex $X$ has infinitely many torsion gaps of the form $\left[k, k+n_{X}-1\right]$, and if $\operatorname{cat}(X) \leq 2$, then $X$ has the rational homotopy type of a wedge of $n_{X}$-spheres.

\section{Proof of Theorem A}

The main tool in this paper is Sullivan's theory of minimal models. A commutative differential graded algebra (CDGA) over the rational number field $Q$ is a differential graded algebra $(A, d)$, where $A=\sum_{p \geq 0} A^{p}, a b=(-1)^{p q} b a$ for $a \in A^{p}$, $b \in A^{q}$, and $d: A^{p} \rightarrow A^{p+1}$ is a derivation. Its cohomology is denoted by $H^{*}(A, d)$. If $V=\sum_{p>0} V^{p}$ is a graded vector space over $Q$ where $V^{p}$ is finite dimensional for all $p$, then we denote by

$$
\bigwedge V=\text { Exterior algebra }\left(V^{\text {odd }}\right) \otimes \text { Symmetric algebra }\left(V^{\text {even }}\right)
$$

the free commutative graded algebra on $V$. A Sullivan minimal model is a CDGA of the form $(\bigwedge V, d)$, where $V^{i}$ is finite dimensional for $i \geq 1$ and $\operatorname{Im} d \subset(\bigwedge V)^{+}$. $(\bigwedge V)^{+}$, where $(\bigwedge V)^{+}$is the ideal generated by $V$. To any simply connected space $X$ s.t. each $H_{i}(X, Q)$ is finite dimensional, $i \geq 2$, Sullivan associates a unique minimal model $(\bigwedge V, d)$, satisfying the following properties:

$$
H(\bigwedge V, d) \cong H^{*}(X, Q) ; \quad V \cong \operatorname{Hom}_{\mathbb{Z}}\left(\pi_{*}(X), Q\right) .
$$

To prove Theorem $\mathrm{A}$, we need two lemmas. Let $(\bigwedge V, d)$ be a minimal model, then the augmentation $\bigwedge V \rightarrow Q$ has a minimal model of the form $(\bigwedge V \otimes \wedge \bar{V}, d) \cong$ $Q$, in which the differential in the quotient model $Q \otimes \bigwedge_{V}(\bigwedge V \otimes \bigwedge \bar{V})=\bigwedge \bar{V}$ is zero and $V^{i} \cong \bar{V}^{i-1}$. Thus each $\bigwedge V \otimes(\bigwedge \bar{V}) \leq r$ is a sub $-(\bigwedge V, d)$ differential model of $\bigwedge V \otimes \bigwedge \bar{V}$. Thus we get a spectral sequence $E_{i}$ with $E_{0}^{p, q}=(\bigwedge V)^{p} \otimes(\bigwedge \bar{V})^{q}$.

Lemma 1. If $V$ is finite dimensional and we let $m=\max \left\{i \mid V^{i} \neq 0\right\}$, then the spectral sequence $E_{i}$ degenerated at $E_{m+1}$, that is, $E_{m+1}=E_{m+2}=\cdots=E_{\infty}$.

Proof of Lemma 1. It is enough to prove by induction on $r$ that the spectral sequence for $\bigwedge V \leq r \otimes\left(\bigwedge \bar{V}^{<r}\right)$ collapses at the $E_{r+1}$-term. The inductive hypothesis then gives the isomorphism

$$
\left(E_{r+1}\left(\bigwedge V^{\leq r+1} \otimes\left(\bigwedge \bar{V}^{\leq r}\right)\right), d_{r+1}\right) \cong\left(E_{r+1}\left(\bigwedge V^{r+1} \otimes\left(\bigwedge \bar{V}^{r}\right)\right), d\right),
$$

with $d(\bar{\nu})=v$. This clearly implies the result.

From Lemma 1, we easily get

Corollary 2. Let $V$ be finite dimensional and $m=\max \left\{i \mid V^{i} \neq 0\right\}$. If $H^{k}(\bigwedge V, d) \neq$ $0, H^{l}(\bigwedge V, d) \neq 0$, and $H^{i}(\bigwedge V, d)=0$ for $k<i<l$, then $l-k \leq m$. 
Proof. If $l-k>m$, then by Lemma $1 E_{\infty}^{l, 0} \neq 0$, which is impossible.

Like homotopy torsion gap, we define homology torsion gap as follows. Let $X$ be a simply connected CW complex, with finite Betti numbers. If $H_{k}(X, Q) \neq 0$, $H_{l}(X, Q) \neq 0$, and $H_{i}(X, Q)=0$ for $k<i<l$, then we call $[k, l]$ a homology torsion gap for $X$.

By Corollary 2, we obtain

Corollary 3. Let $X$ be a simply connected rational elliptic $C W$ complex and $m=$ $\max \left\{i \mid \pi_{i}(X) \otimes Q \neq 0\right\}$. If $[k, l]$ is a homology torsion gap for $X$, then $l-k \leq m$.

Remark. Corollary 3 is a dual form of Theorem 1 (Halperin), in the sense of Eckman-Hilton duality; furthermore, they are equivalent.

We also need the following lemma, which easily followed from S. Halperin and G. Levin [5].

Lemma 2. Let $X$ be a simply connected finite $C W$ complex with $(\bigwedge V, d)$ as minimal model. Denote by $J$ the differential ideal of $(\bigwedge V, d)$ defined by $J=(\bigwedge V)^{>n_{X}} \oplus$ $W$, where $W$ is the complement in $(\bigwedge V)^{n_{X}}$ of the cocycles. Let $(\bigwedge V \leq k, d)$ be a submodel of $(\bigwedge V, d)$ and $\bigwedge V^{\leq k} \otimes \bigwedge U$ be a minimal of $\bigwedge V^{\leq k} \rightarrow Q$. Then the minimal model of $(\bigwedge V \leq k \otimes \bigwedge U) \otimes \bigwedge_{V \leq k} \wedge V / J=\bigwedge U \otimes \wedge V / J$ has the same minimal model of $X(k)$, the $k$-connected covering of $X$.

A CDGA morphism, $\phi$, is a quism if $H(\phi)$ is an isomorphism. The equivalence class of a CDGA (under the equivalence relation generated by quisms) is called its homotopy type. A CDGA is said to be a wedge of spheres if it has the homotopy type of a connected CDGA $H$ with differential zero and satisfies $H^{+} \cdot H^{+}=0$.

Now we prove Theorem A.

Proof of Theorem $A$. For $\bigwedge V^{\leq k} \otimes \wedge U$ and $\Lambda U \otimes \wedge V / J$, we have two spectral sequences $\bar{E}_{m}, E_{m}$ respectively (filtered by the degree of $\Lambda U$ ) and morphism $\phi_{m}$ : $\bar{E}_{m} \rightarrow E_{m}$ induced by $\bigwedge V^{\leq k} \rightarrow \bigwedge V^{\leq k} / J$. Because $\bar{E}_{2}^{i, *} \rightarrow E_{2}^{i, *}$ is identical for $i<k+n_{X}, \phi_{k+1}: \bar{E}_{k+1}^{i, *} \rightarrow E_{k+1}^{i, *}$ is an isomorphism for $i \leq n_{X}-1$, while $\bar{E}_{k+1}^{i, *}=0$ for all $(i, *) \neq(0,0)$. Thus $E_{k+1}^{i, *} \neq 0$ only for $i=n_{X}$ or $(i, *)=(0,0)$. Therefore, we can choose the representatives of $H^{+}(\bigwedge U \otimes \wedge V / J)$ from $\bigwedge U \otimes(\bigwedge V / J)^{n_{X}}$. Obviously their product is zero; thus $\bigwedge U \otimes \wedge V / J$ is a wedge of spheres.

By Lemma 2, we know that the minimal model of $X(k)$ is a wedge of spheres; thus, we get Theorem A.

\section{THE SECOND MAIN THEOREM}

Before proving Theorem B, we recall some definitions. In [2], Felix and Halperin define the rational L.S. category, $\operatorname{Cat}_{0}(X)$, via the Sullivan minimal model $(\bigwedge V, d)$ for $X$, $\operatorname{Cat}_{0}(X) \leq m$ if and only if in the diagram

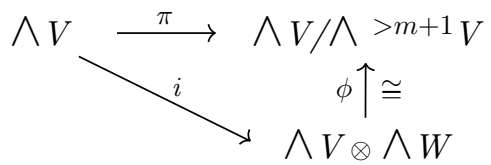

there exists $r$, so that $r i$ is identity, where $\pi$ is the projection and $\phi$ is a quism. When $X$ is a simply connected space with finite Betti numbers, they prove $\operatorname{Cat}_{0}(X)=$ $\operatorname{Cat}\left(X_{Q}\right)$. 
Let $L$ be a graded Lie algebra. Then the radical of $L, \operatorname{rad} L$, is the sum of all resoluble ideals of $L$.

$$
\begin{aligned}
\text { gl. } \operatorname{dim} L & =\text { gl. } \operatorname{dim} U L=\sup \left\{i \mid \operatorname{Ext}_{U L}^{i}(Q, Q) \neq 0\right\}, \\
\operatorname{depth} L & =\operatorname{depth} U L=\inf \left\{i \mid \operatorname{Ext}_{U L}^{i}(Q, U L) \neq 0\right\} .
\end{aligned}
$$

We need the following theorems from Felix et al. [4] and Felix [1].

Theorem 2. Let $X$ be a simply connected space with finite Betti numbers. Then

(1) depth $H_{*}(\Omega X, Q) \leq \operatorname{Cat}_{0}(X) \leq \operatorname{gl} . \operatorname{dim} H_{*}(\Omega X, Q)$,

(2) if depth $H_{*}(\Omega X, Q)=\operatorname{Cat}_{0}(X)$, then

$$
\operatorname{Cat}_{0}(X)=\text { gl. } \operatorname{dim} H_{*}(\Omega X, Q) .
$$

Theorem 3. Let $L$ be a graded Lie algebra and $\operatorname{depth} L=m<\infty$. Then

(1) $\operatorname{dim}(\operatorname{rad} L)_{\text {even }} \leq m$,

(2) $\operatorname{rad} L$ is finite dimensional,

(3) if $\operatorname{dim}(\operatorname{rad} L)_{\text {even }}=m$, then $L=\operatorname{rad} L$.

Theorem 4. If $\operatorname{Cat}_{0}(X) \leq 2$ and gl. $\operatorname{dim} H_{*}(\Omega X, Q)=2$, then $X$ is a coformal space, that is, if $(\bigwedge V, d)$ is the Sullivan minimal model, then $d=d_{2}$.

Proof of Theorem B. First, $L=\pi_{*}(\Omega X) \otimes Q$ is a graded Lie algebra by the Samelson Product. We claim that depth $L \leq 1$. Otherwise depth $=2=\operatorname{Cat}_{0}(X)$; hence, by Theorem 2, gl. $\operatorname{dim} L=2$. According to Theorem $4, X$ is a coformal space; hence, the Sullivan minimal model has the form $(\bigwedge V, d), d=d_{2}$. Now let $k_{0}=\min \left\{k \mid\left[k-n_{X}+1, k\right]\right.$ is a torsion gap for $\left.X\right\}$ and take $\alpha \in V$ s.t. $|\alpha|>2 k_{0}$. Then

$$
d \alpha=\beta_{1} \gamma_{1}+\beta_{2} \gamma_{2}+\cdots+\beta_{m} \gamma_{m} .
$$

We may suppose $\left|\beta_{1}\right|=\max \left\{\left|\beta_{i}\right|,\left|\gamma_{i}\right|\right\}$. Then $\left|\beta_{1}\right| \geq \frac{|\alpha|}{2}>k_{0}$; thus $\left|\beta_{1}\right| \equiv 1$ $\left(\bmod n_{X}-1\right)$ (by Corollary 1$)$. We can suppose that $d \alpha=\beta_{1} \gamma+\cdots \quad$ (the other terms having no $\left.\beta_{1}\right)$ in view of $d d \alpha=0, d \gamma \cdot \beta_{1}=0$, hence $d \gamma=0$ and $|\gamma|=n_{X}$ because $\left|\beta_{1}\right| \equiv|\alpha| \equiv 1 \bmod n_{X}-1$. Therefore $X_{Q} \cong\left(S^{n_{X}} \vee Y\right)_{Q}$, but this is impossible (because of depth $\left.\pi_{*}\left(\Omega\left(S^{n_{X}} \vee Y\right)\right) \otimes Q\right)=1$ ). So we have $\operatorname{depth} L \leq 1$.

Next, we claim that if $x \in \pi_{*}(X) \otimes Q \cong \pi_{*-1}(\Omega X) \otimes Q$ and $|x| \neq 1\left(\bmod n_{X}-1\right)$, then $x \in \operatorname{rad} L$.

In fact, we denote by $I$ the Lie ideal generated by $x$; then we say $I_{\geq k_{0}}=0$. Otherwise there is some $\alpha \neq 0, \alpha \in I_{\geq k_{0}}$. Since $I_{\geq k_{0}}$ is a free Lie algebra, there is some $\omega \in I_{\geq k_{0}}$ s.t. $[\alpha, \omega] \neq 0$. We write $\alpha=\sum_{i}\left[\beta_{1 i}, \beta_{2 i}\right]$, with $\beta_{1 i} \in I$. The Jacobi identity implies that there is some $i$ and some $u \in I_{\geq k_{0}}$, s.t. $\left[\beta_{1 i}, u\right] \neq 0$. Clearly $\left|\beta_{1 i}\right|<|\alpha|$. We continue the same procedure with $\beta_{1 i}$ in place of $\alpha$. After finitely many steps, we get $[x, u] \neq 0$, where $|u| \equiv 1\left(\bmod n_{X}-1\right)$ and $|u|>k_{0}$, but $\pi_{|u|+|X|}(X) \otimes Q=0$. This is a contradiction, so $I_{\geq k_{0}}=0$, hence $x \in \operatorname{rad} L$.

We know depth $L=1$, by Theorem 3 , and $(\operatorname{rad} L)_{\text {even }}=0$, hence $\pi_{2 k+1}(X) \otimes Q=$ 0 if $2 k+1 \neq 1\left(\bmod n_{X}-1\right)$. If $n_{X}$ is odd, then we must have some $\alpha \in \pi_{n_{X}}(X) \otimes Q$, and $d \alpha=0$, so $X_{Q}=\left(S^{n_{X}} \vee Y\right)_{Q}$. If $Y_{Q} \neq \bigvee S^{n_{X}}$, by the following Lemma $3, X$ has no torsion gap of the form $\left[k, k+n_{X}-1\right]$, so that $Y_{Q} \cong \bigvee S^{n_{X}}$, and $X$ has the rational homotopy type of a wedge of $n_{X}$-spheres. If $n_{X}$ is even and if $m<n_{X}$, then $\bigwedge V^{\leq n_{X}}$ is a polynomial algebra and $d V \leq n_{X}=0$. Let $\alpha \in \pi_{m}(X) \otimes Q$, where $m=\min \left\{k \mid \pi_{k}(x) \otimes Q \neq 0\right\}$, and $\beta \in(\bigwedge V)^{n_{X}}$ representing a cohomology of $H^{n_{X}}(\bigwedge V, d)$. Then $d(\alpha \beta)=0,|\alpha \beta|=m+n_{X}<2 n_{X}-1$, so that $\alpha \beta$ cannot be a 
coboundary; hence $H^{m+n_{X}}(\bigwedge V, d) \neq 0$. This is in contradiction with the definition of $n_{X}$. So $m=n_{X}$, hence, by the rational Hurewicz Theorem, $X$ has the rational homotopy type of a wedge of $n_{X}$-spheres.

Lemma 3. If $X=S^{n_{X}} \vee Y$ and $Y_{Q} \neq\left(\bigvee S^{n_{X}}\right)_{Q}$, then $X$ has no torsion gap of the form $\left[k, k+n_{X}-1\right]$.

Proof. As a Lie algebra, $\pi_{*}(\Omega X) \otimes Q$ is the free product of $\pi_{*}\left(S^{n_{X}}\right) \otimes Q$ and $\pi_{*}(Y) \otimes Q$. It is easy to show that $X$ has no torsion gap of the form $\left[k, k+n_{X}-1\right]$.

\section{ACKNOWLEDGMENT}

Finally, the author would like to acknowledge Professor Shen Xinyao for his helpful comments, and to also acknowledge the referee for offering a corrective proof of Lemma 1 and other useful remarks.

\section{REFERENCES}

[1] Y. Félix, La dichotomie elliptique-hyperbolique en homotopie rationnelle, Asterisque 176 (1989). MR 91c:55016

[2] Y. Félix and S. Halperin, Rational L.S. category and its application, Trans. Amer. Math. Soc. 273 (1982), 1-37. MR 84h:55011

[3] Y. Félix, S. Halperin, C. Jacobson, C. Löfwall, and J.-C. Thomas, The radical of the homotopy Lie algebra, Amer. J. Math. 110 (1988), 301-322. MR 89d:55029

[4] S. Halperin, Torsion gaps in the homotopy of finite complexes. II, Topology 30 (1991), 471478. MR 92f:55014

[5] S. Halperin and G. Levin, High skeleta of $C W$-complexes, Lecture Notes in Math, vol. 1183, Springer-Verlag, Berlin, 1986, pp. 211-217. MR 87m:55013

[6] H. Huang, On a conjecture of S. Halperin, Northeast Math. J. 10 (1994), 517-524.

Institute of Mathematics, Academia Sinica, Beijing 100080, People's Republic of CHINA

Current address: Ph.D. Program in Mathematics, Graduate Center, CUNY, 33 W 42 St., New York, New York 10036

E-mail address: hhuang@email.gc.cuny.edu 